\title{
EL DERECHO A BENEFICIARSE DE LOS AVANCES CIENTÍFICOS Y LOS LÍMITES A LA DISCRECIONALIDAD ESTATAL: LA SALUD REPRODUCTIVA EN AMÉRICA LATINA ${ }^{1}$ \\ Agustina Ramón Michel $^{2}$ \\ Sonia Ariza Navarrete ${ }^{3}$
}

Resumen: Las mujeres de países desarrollados rara vez mueren de hemorragia postparto y casi nunca por un aborto, las mujeres en Latinoamérica sí. La mayoría de estas muertes son evitables, y el misoprostol y la mifepristona pueden ayudar a prevenirlas. El misoprostol tiene varios usos obstétricos, incluido el tratamiento de la hemorragia postparto y el aborto incompleto. La mifepristona tiene como principal uso la interrupción del embarazo. Estas ventajas explican el aumento de su uso por parte de profesionales de la salud así como de mujeres, transformando las experiencias del aborto y disminuyendo los abortos inseguros. Sin embargo, pocos países latinoamericanos tienen registrados estos medicamentos para usos obstétricos pese a que son fármacos seguros, eficaces, costo-efectivos, de alta aceptabilidad, y están incluidos en la lista de medicamentos esenciales de la Organización Mundial de la Salud (OMS). Este tipo de comportamiento estatal, indolente y sesgado en la evaluación de estos medicamentos, refleja más posiciones morales particulares, conveniencias políticas, desprecio hacia la salud de las mujeres, y estigmatizaciones hacia el aborto, que posiciones basadas en evidencia científica, en las experiencias de mujeres $\mathrm{y}$ profesionales, $\mathrm{y}$ en las obligaciones derivadas de los derechos de las mujeres.

1 Este artículo es una versión modificada del documento "Entre la indolencia y el sesgo: el derecho de las mujeres a beneficiarse de los avances científicos en materia reproductiva", Consorcio Latinoamericano Contra el Aborto Inseguro (CLACAI), febrero de 2017.

2 Abogada. Profesora de la Facultad de Derecho de la Universidad de Palermo e investigadora adjunta del Centro de Estudios de Estado y Sociedad. Maestría en derecho con especialización en América Latina por la University of Texas, Austin, y Especialización en derechos de las mujeres por la Universidad de Chile.

3 Abogada. Maestría en Derecho comparado en el Instituto Universitario Europeo (Italia). Candidata doctoral en Derecho por la Universidad de Palermo. 
En este artículo argumentamos que esta situación constituye una forma de discriminación principalmente hacia las mujeres en el ejercicio de su derecho a beneficiarse de los avances científicos, entre otros derechos. El margen de discreción de los Estados para adoptar políticas y garantizar el ejercicio de los derechos no es ilimitado, y la política de medicamentos en salud reproductiva no es la excepción; los gobiernos deben poder justificar públicamente sus elecciones y prioridades de política.

Palabras claves: Derecho a gozar de los avances científicos. Salud reproductiva. Misoprostol. Mifepristona. América Latina.

Abstract: Women from developed countries rarely die from postpartum hemorrhage and almost never from abortion, whereas women in Latin America do die. Most of these deaths are preventable, and misoprostol and mifepristone can help to prevent them. Misoprostol has several obstetric uses, including treatment of postpartum hemorrhage and incomplete abortion. Mifepristone has as its primary use the interruption of pregnancy. The advantages of these drugs have led to an increased use by health professionals and women, transforming abortion experiences and reducing unsafe abortions. However, most Latin American countries have not registered these drugs for obstetric uses, despite the fact that they are safe, effective, costeffective and with high acceptability. Both drugs are included in the list of essential medicines of the World Health Organization (WHO). The indolent and biased State's behavior in the evaluation of these drugs reflects particular moral positions, political bias and disdain for women together with stigmatization towards abortion. In this paper, we argue that this situation constitutes a form of discrimination against women in the exercise of their right to benefit from scientific advances, together with the violation of the right to life, health, among other rights. The discretion of States to adopt policies and guarantee the exercise of rights is not unlimited. Reproductive health policy is no exception. Governments should be obliged to publicly justify their choices and policy priorities. 
Keywords: Right to enjoy scientific advances. Reproductive health. Misoprostol Mifepristone. Latin America.

\section{INTRODUCCIÓN}

Aun hoy, la hemorragia postparto y el aborto practicado en condiciones de inseguridad son dos de las principales causas de muerte asociadas al embarazo en América Latina, pese a que existen tecnologías sencillas para prevenirlas o tratarlas; el misoprostol y la mifepristona son dos de esas tecnologías. Ambos fármacos tienen varios usos en salud reproductiva (el misoprostol sirve para el tratamiento de la hemorragia postparto, del postaborto y parto) figuran en la lista de medicamentos esenciales de la Organización Mundial de la Salud (OMS) desde hace más de una década y combinados constituyen uno de los métodos más seguros y eficaces para interrumpir el embarazo.

Estos medicamentos han transformado la práctica y la experiencia

\footnotetext{
${ }^{4}$ En este artículo nos referiremos a las cis-mujeres dado que estadísticamente representan la mayoría de las personas que requieren servicios de aborto, sin
}

de las mujeres que abortan y redujeron el aborto inseguro. ${ }^{4}$ Sin embargo, las agencias y políticas de medicamentos de América Latina han quedado rezagadas en sus respuestas a estos cambios de tecnología reproductiva.

El misoprostol para usos obstétricos ha sido registrado en más de noventa países y la mifepristona en más de cincuenta, pero en América Latina la mayoría de los Estados ha permanecido inactiva $o$ ha demorado injustificadamente u obstruido el registro de estos medicamentos. Estas omisiones han contribuido a restringir indebidamente su uso, aumentar su costo (es la región donde el misoprostol se vende a precios más altos) y exponer a mujeres a falsificaciones o dosis inadecuadas. Esta situación continua una tradición de indolencia y discriminación respecto a medicamentos y tecnologías asociadas a la salud sexual y reproductiva de las mujeres.

Aunque generalmente los gobiernos alegan que se trata de consecuencias propias del diseño del

desconocer que existen personas con otras identidades de género que pueden y tienen derecho a requerir abortos. 
sistema de registro y aprobación de medicamentos, basado en la pasividad y neutralidad del Estado, hay buenos ejemplos de situaciones donde se ha dejado de lado estos principios por razones de reducción de costos, priorización y acceso especial de medicamentos en vías de garantizar el derecho a la salud de las mujeres. No ha sido el caso del misoprostol y la mifepristona.

En este artículo argumentamos que la actitud de la mayoría de las agencias sanitarias y de medicamentos latinoamericanos viola el derecho a beneficiarse de los avances científicos de las mujeres, y por tanto vulneración en el ejercicio de este derecho tan vinculado a los derechos a la vida, a la salud y la autonomía, entre otros. Más aun, se discrimina a las mujeres en el goce del derecho a beneficiarse de los avances científicos en salud reproductiva.

El derecho a beneficiarse de los avances científicos fue reconocido por primera vez en 1948 y reafirmado luego en diversos instrumentos y órganos internacionales. En materia de salud, el progreso científico es clave; el acceso a los servicios de buena calidad, incluido insumos y desarrollos científicos, suele ser la única forma de realizar el derecho a la salud.

Las políticas de salud incluidas las de medicamentos son delineadas e implementadas según la evaluación de funcionarios públicos y expertas gubernamentales. Pero esta discrecionalidad tiene límites. Primero, porque las políticas deben encaminarse a realizar los derechos. Segundo, porque los derechos constitucionales incluidos buena parte de los derechos humanos (que, además, tienen la más alta jerarquía en los sistemas jurídicos latinoamericanos), ponen límites a esa discrecionalidad, y permiten distinguir entre acciones y omisiones constitucionales o inconstitucionales. Tercero, porque los actores estatales deben poder justificar con razones públicas sus decisiones.

Por otro lado, todos los países latinoamericanos están comprometidos jurídicamente a brindar atención en salud materna. Por otro lado, la mayoría de los sistemas jurídicos de la región contemplan causales legales para abortar (usualmente riesgo para la vida y la salud), y los servicios de salud, 


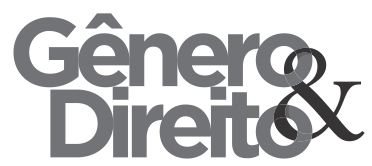

independientemente del estatus jurídico del aborto, están obligados a atender las complicaciones por aborto pues son servicios de emergencia obstétrica. Además, como ha quedado establecido desde la década de 1990, la mortalidad y morbilidad materna constituyen un asunto de derechos humanos, violaciones al derecho a la vida y a la salud, entre otros derechos.

Lo que se observa regionalmente es un escenario distinto al normativo: los Estados se mantienen pasivos o incluso reactivos a la aprobación del misoprostol y mifepristona pese a tratarse de medicamentos seguros, efectivos, de alto costo-efectividad que pueden salvar la vida y mejorar la salud materna $\mathrm{y}$ reproductiva de las mujeres.

1. CONTEXTO: LA MORBIMORTALIDAD POR CAUSAS ASOCIADAS AL EMBARAZO Y ABORTO EN AMÉRICA LATINA

La OMS estima que entre el $88 \%$ y el $98 \%$ de las muertes maternas son evitables (OMS, 1986) con
Ciódico do Núcleo de Estudos e Pesquisas sobre Gênero e Direito Centro de Ciências Jurídicas - Universidade Federal da Paraíba V. 6 - No 03 - Ano 2017 (Spanish Edition) 244 intervenciones costo-efectivas y un adecuado respecto a los derechos de las mujeres. Así, el derecho a la información sobre síntomas de emergencia obstétrica aumenta las posibilidades de que una mujer identifique y acuda a tiempo al centro de salud; el acceso efectivo a anticonceptivos permite un mayor control personal sobre la reproducción (Ramos et al., 2004).

Las causas más frecuentes de mortalidad materna en América Latina son la hipertensión inducida por el embarazo, las hemorragias, las complicaciones por aborto inseguro y el parto obstruido (Romero y Ramón Michel, 2014). La mayoría de ellas son causas evitables. La evidencia construida desde la década del 90 ha mostrado consistentemente la eficacia del misoprostol y mifepristona, especialmente en la disminución del aborto inseguro y el control de hemorragias obstétricas.

Aunque la razón de mortalidad materna en la región ha disminuido, existen dramáticas diferencias entre países, la tasa en el Caribe es de $190 \mathrm{x}$ $100.000 \mathrm{NV}$, y la del resto de América Latina es 72 x 100.000 (Romero y Ramón 
Michel, 2014) y al interior de estos, en áreas con altas proporciones de población Desde hace más de 20 años la indígena y de mujeres negras los riesgos de muerte materna superan ampliamente los del promedio nacional (UNICEF, 2011); y es la primera causa de muerte de mujeres adolescentes de entre quince y diecinueve años (Romero y Ramón Michel, 2014).

En la región, hay cerca de 4,6 millones de abortos inseguros anuales, con las tasas de incidencia más altas del mundo (44 abortos por cada mil mujeres de 15 a 44 años de edad) (Guttmacher, 2016). De esas mujeres, 750.000 acuden todos los años a los servicios públicos de salud por complicaciones de abortos inseguros. Finalmente, alrededor del $12 \%$ de las muertes maternas se deben a complicaciones de aborto inseguros (OMS, 2008).

\section{BREVE DESCRIPCIÓN SOBRE MISOPROSTOL MIFEPRISTONA5}

\section{Misoprostol}

OMS considera al misoprostol un medicamento esencial debido a su impacto en la protección de la salud de las mujeres (OMS, 2005). Es un análogo de la prostaglandina E1 (PgE1), indicado originalmente para el tratamiento de la úlcera péptica (FLASOG, 2013). Sirve para el tratamiento de la hemorragia postparto, principal causa de mortalidad materna en el mundo (FIGO, 2014). Es también un método sencillo, seguro, no invasivo $\mathrm{y}$ de bajo costo para el tratamiento del aborto incompleto y constituye, solo o combinado con mifepristona, un régimen de aborto eficaz y seguro (FLASOG, 2013). Asimismo, está probada su eficacia para la maduración cervical, la inducción del parto y la evacuación tras la muerte fetal intrauterina o el embarazo anembrionado o retenido (FLASOG, 2013). Puede emplearse para la dilatación del cuello uterino, para la inserción del dispositivo intrauterino o de una histeroscopía (FLASOG, 2007). Respecto al aborto, el

5 Agradecemos la recomendación de materiales de Laura Gil de Oriéntame (Colombia) para la elaboración de esta sección. 
esquema de misoprostol tiene hasta un 90\% de eficacia durante las primeras nueve semanas de gestación y alrededor de $85 \%$ después (OMS, 2012). Y dado que los riesgos asociados a su uso son muy bajos, está recomendado en todos los niveles de atención hasta la semana diez/doce según el esquema y las condiciones de cada mujer (OMS 2015).

Desde los años 80 se viene observando el impacto de estos fármacos en la disminución de la mortalidad materna por su alta efectividad en los usos recién mencionados (Coêlho, et al., 1993). Sin embargo, en la mayoría de América Latina, la disponibilidad del misoprostol aún está restringida, lo que hace su uso costoso en varios sentidos, tanto para las mujeres como para profesionales de la salud y el sistema de salud.

El siguiente mapa muestra las aprobaciones del misoprostol en el mundo.

\section{Gráfico 1. Mapa de la aprobación del misoprostol por causales en el mundo hasta el}

\section{3}

\section{Global Misoprostol Registration by Indication}

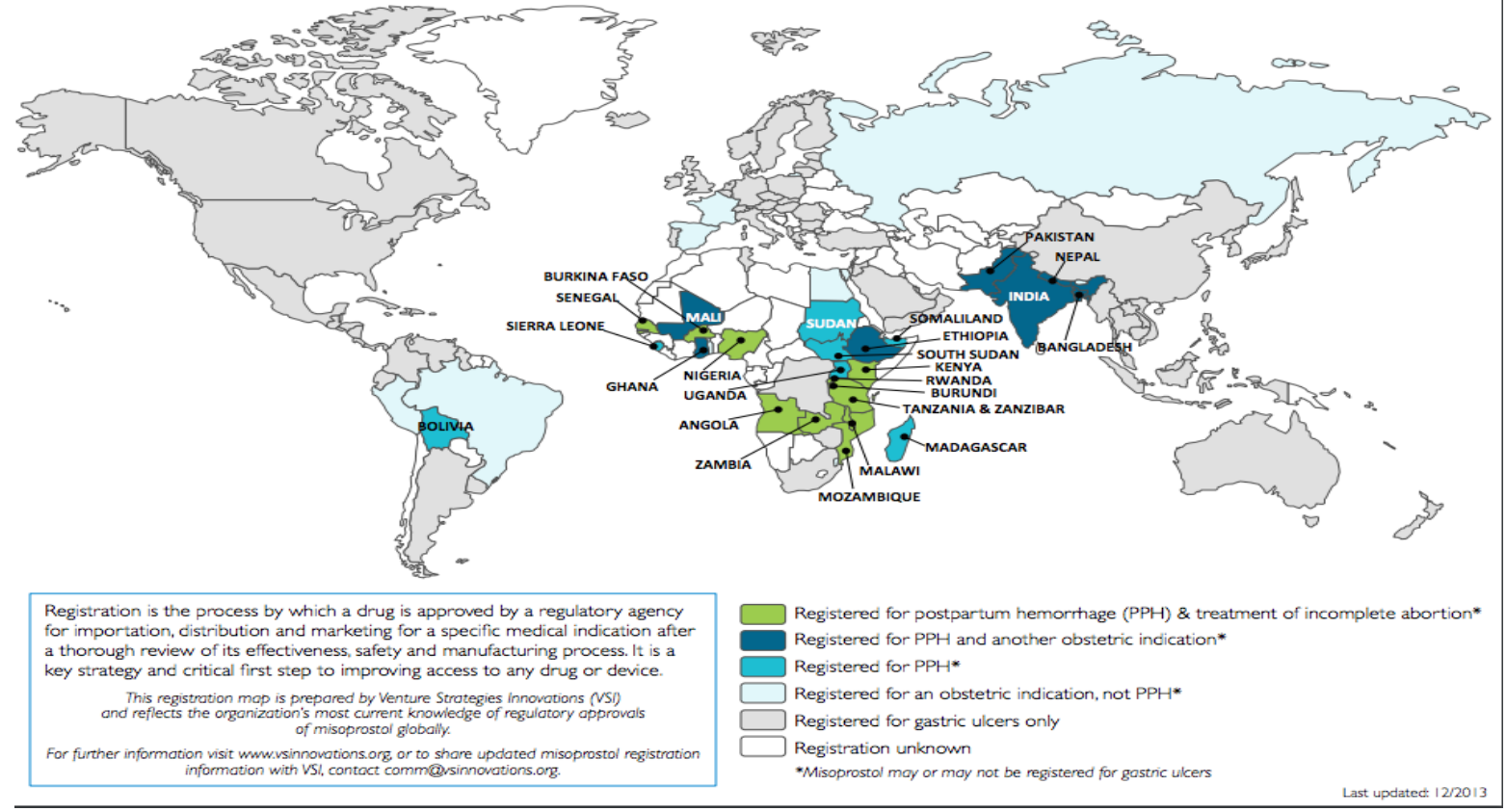

Fuente: Gynuity. Venture Strategies Innovations, 2013. 
Según muestra el mapa, noventa países registraron el misoprostol para al menos uno de sus usos obstétricos. En la mayoría de los países latinoamericanos, en cambio, el misoprostol no está registrado para usos obstétricos, o su aprobación o uso están restringidos (CLACAI- y FLASOG, 2013). Solo doce países de la región tienen aprobado al menos un uso obstétrico del misoprostol (Távara y Chávez, 2013). Con excepción de Colombia, Costa Rica, Cuba, Ciudad de México y Uruguay no hay registros para su uso en aborto a pesar de existir indicaciones legales para el aborto. Por su parte, Argentina, Brasil, Chile y Perú aprobaron algunos usos obstétricos pero no para el manejo de la hemorragia postparto y el aborto, dos de sus usos más comunes (Távara y Chávez, 2013). Y Bolivia lo registró sólo para casos de emergencia de hemorragia posparto.

Dada esta falta de registro para varias indicaciones obstétricas, su disponibilidad en dosis/presentaciones inadecuadas para abortar y restricciones en las regulaciones de uso, ${ }^{6}$ en muchos

6 Las experiencias regulatorias en el mundo reflejan un atraso respecto de la evidencia científica que respalda los múltiples usos obstétricos del misoprostol. Es así como la Agencia Europea de países es usada off label y se lo obtiene a un costo económico más elevado sumando a las dificultades y azares para dar con la medicación (CLACAI, 2015).

\section{Mifepristona}

Desde 2005, la mifepristona es considerada un medicamento esencial por la OMS. También conocida en su origen como RU486, es un antiprogestágeno y su uso más difundido es para la interrupción del embarazo (Winikoff y Sheldon, 2012; Boonstra, 2013), lo que ha sometido su aprobación, disponibilidad y accesibilidad a procesos contenciosos, lentos y extremadamente politizados en varios países del mundo (Berer, 1993; Clarke y Montini, 1993; Hogan, 2010).

La mifepristona junto con el misoprostol son el estándar de oro para la interrupción del embarazo, pues presenta altísima efectividad y pocos efectos secundarios (Winikoff y Sheldon, 2012). De la misma forma que el misoprostol solo, el régimen combinado puede

Medicamentos ha aprobado el uso del misoprostol para el manejo de la hemorragia postparto, pero la mayoría de los países europeos mantienen únicamente la aprobación para manejo de las úlceras pépticas. 
emplearse de manera ambulatoria hasta las semanas 9-12 de gestación por lo menos (OMS, 2012; Faundes y Figo, 2011), y es especialmente efectivo y seguro para los de segundo trimestre dentro de los establecimientos sanitarios
(Federación Colombiana de Ginecología y Obstetricia, 2013). El siguiente mapa muestra la distribución geográfica y temporal de la aprobación de la mifepristona en el mundo.

Gráfico 2. Mapa de aprobación de mifepristona en el mundo hasta 2014

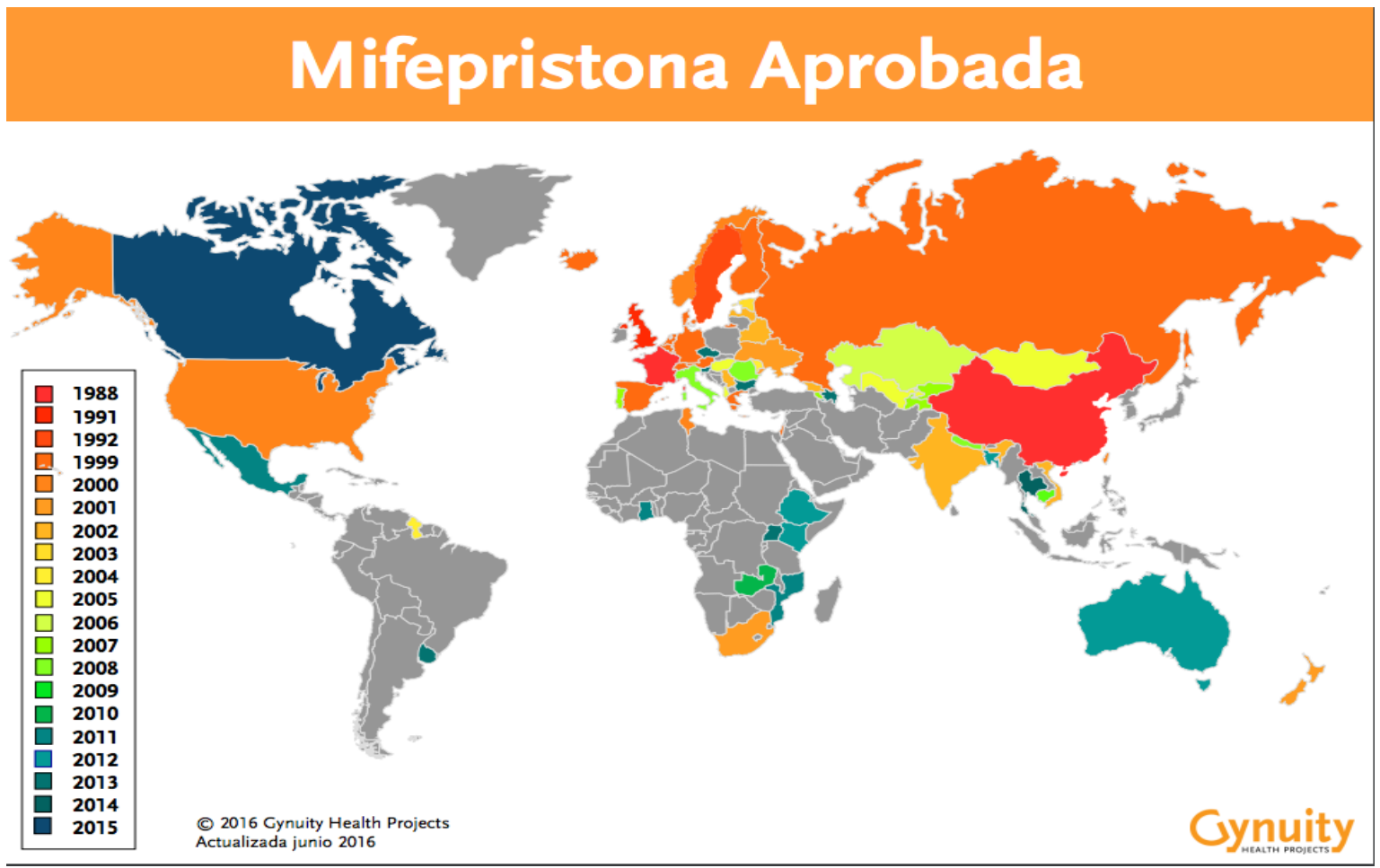

Fuente: Gynuity Health Projects, 2014.

Como se observa en el gráfico, un mayor número de países en vías de desarrollo avanzaron en la aprobación de este medicamento. No obstante, América Latina y África continúan siendo las regiones con menor proporción de aprobaciones. En América Latina, la mifepristona está aprobada sólo en Guyana, México, Uruguay y en Colombia.

3. CONSECUENCIAS PRÁCTICAS DE LA FALTA DE APROBACIÓN Y OTRAS BARRERAS DE 


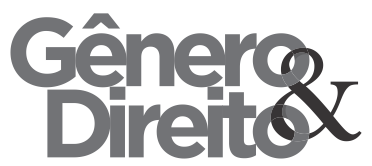

DISPONIBILIDAD

\section{ACCESIBILIDAD}

La falta de registro del misoprostol para la mayoría de los usos obstétricos apareja una serie de consecuencias gravosas para la vida, salud y bienestar de las mujeres, así como dificultades para los profesionales de la salud y el sistema de salud en general.

En primer lugar, los efectos por las formas de uso. Dado que en la mayoría de los países latinoamericanos está aprobado para el tratamiento de la úlcera gástrica, las presentaciones disponibles son de administración oral y muchas vienen combinadas con un analgésico no esteroideo, en general Diclofenac. Cuando estos comprimidos son empleados para usos obstétricos, especialmente por vía vaginal, son menos apropiados pues la presentación recomendada para estos usos son los óvulos o comprimidos solubles, disponibles en Europa y algunos lugares de la región.

En segundo lugar, la distribución también está restringida por las regulaciones que fijan requisitos injustificados: venta con receta
Ciódico do Núcleo de Estudos e Pesquisas sobre Gênero e Direito

Centro de Ciências Jurídicas - Universidade Federal da Paraíba

V. 6 - No 03 - Ano 2017 (Spanish Edition) 249

Y archivada, distribución intrahospitalaria exclusiva, prohibición de publicidad, restricciones para su uso ambulatorio. En América Latina, 28 regulaciones nacionales exigen la receta médica (Távara y Chávez, 2013).

En tercer lugar, las mujeres que usan este método de manera ambulatoria usualmente recurren al circuito clandestino para adquirir el medicamento debido a las restricciones mencionadas, o por la falta de aprobación para esta indicación o su falta de disponibilidad en los servicios de salud. De este modo, quienes acceden a este mercado, ya sea en farmacias o por internet, no sólo pagan sobreprecio por el misoprostol sino que a veces reciben fármacos incorrectos, comprimidos sueltos (que impide verificar vencimiento y compromete su calidad), dosis menores que las recomendadas, o se ven obligadas a correr riesgos por lugares donde se los venden (ej. expendios de drogas psicoactivas en el caso de Argentina; Lesbianas y Feministas por la Discriminación del Aborto, 2015).

Por su parte, la falta de aprobación de la mifepristona les quita a mujeres y profesionales de la salud una de 
las formas más seguras, efectivas para interrumpir el embarazo. La mifepristona combinada con misoprostol puede emplearse de forma segura fuera de los servicios de salud hasta la semana diez de gestación y ayuda a que los servicios de salud primarios también puedan brindar atención de aborto legal. Por otro lado, la mifepristona es una aliada clave para los abortos después del primer trimestre, que constituyen una proporción menor en los países con amplio acceso al aborto legal pero que aumenta en países con regulaciones restrictivas o barreras fácticas de todo tipo que generan demoras en el acceso, como los países latinoamericanos (Scot yWeitz, 2009; Harris y Grossman, 2011).

En efecto, pese a que en la mayoría de los países de la región el aborto está despenalizado bajo un modelo de causales, la existencia de la legalidad es aún una construcción frágil ${ }^{7}$ y en muchos lugares de la región lo que aún rige en la práctica es una penalización absoluta que se manifiesta sobre todo en la inaccesibilidad a los servicios de salud pero también en la persecución penal de mujeres (Bergallo, 2014; Centro de Derechos Reproductivos, 2015). De todos modos, incluso en lugares con buen acceso, los abortos de segundo trimestre son inevitables, pues muchas de las patologías del feto (ej.: anencefalia) o de las mujeres embarazadas se desarrollan o son diagnosticadas durante ese período. Además, muchas niñas y adolescentes y mujeres adultas víctimas de violencia (domestica, sexual, institucional, etc.) llegan a los servicios de salud luego de pasadas las primeras semanas de embarazo (Kiley, et al, 2010).

Finalmente, la falta de aprobación y disponibilidad de estos medicamentos enfrenta a muchxs profesionales de la salud a dilemas éticos $\mathrm{y}$ al incumplimiento de sus deberes profesionales. La garantía del derecho a la salud que recae sobre los sistemas de salud y en últimas sobre lxs profesionales incluye brindar el mejor tratamiento posible; no disponer de misoprostol o mifepristona les obliga a ofrecer tratamientos sub-óptimos (ej. dejándoles

\footnotetext{
${ }^{7}$ En jurisdicciones y servicios de salud de Argentina, Bolivia, Brasil, Colombia, Perú, México y Uruguay sobre todo.
} 
el legrado como única opción, cuando no tienen entrenamiento en aspiración endouterina) o incluso la negación del servicio o la derivación argumentando falta de recursos.

Pese a todas las barreras existentes, tanto mujeres como profesionales de la salud han encontrado formas de acceder al misoprostol $\mathrm{y}$, en menor medida, a la mifepristona. Grupos de mujeres se han organizado para difundir información sobre cómo abortar con misoprostol y acompañar a otras mujeres (Zamberlin et al., 2012). ${ }^{8}$ Asimismo, el misoprostol es un medicamento de uso común para ginecobstétras latinoamericanxs; según reportan Távara et al. lxs profesionales usan regularme el Misoprostol "para la evacuación uterina en casos de feto muerto intra-útero, en abortos retenidos y en la inducción del parto. A pesar de no estar normado, su uso en ginecología y obstetricia en muchos países de la región está muy difundido, explicándose su popularidad porque es barato,

8 En distintos países grupos de mujeres se han organizado para acompañar a otras mujeres a abortar de manera segura con medicamentos. Perú (ej. Colectiva por la Libre Información para las Mujeres); Ecuador (ej. Jóvenes por despenalización del aborto); Argentina (ej. Feministas y Lesbianas por la termoestable y efectivo para provocar contracciones uterinas" (Távara, et al., 2008).

\section{EL DERECHO A BENEFICIARSE DE LOS AVANCES CIENTÍFICOS}

De modo cada vez más evidente, la ciencia tiene un impacto en los derechos humanos tanto como factor contribuyente como amenazante. Sin embargo el desarrollo de los derechos y directrices para la interacción entre ellos es todavía incipiente.

Tras la Segunda Guerra Mundial, aparece con mayor fuerza la necesidad de garantizar el goce de los avances científicos, en un clima de preocupación por las aterradoras investigaciones y sus aplicaciones. Ya para ese momento, a la sombra del desarrollo de la energía nuclear y los experimentos científicos del tercer Reich, era claro que la pretensión de neutralidad de la ciencia era inalcanzable, una fantasía que duro poco, y que debían

Descriminalización del Aborto; Socorristas); Chile (ej. Red de Lesbianas y Feministas por el Derecho a la Información); Uruguay (ej. Mujeres en el Horno); México (ej. Las libres); Venezuela (ej. Línea aborto: información segura). 


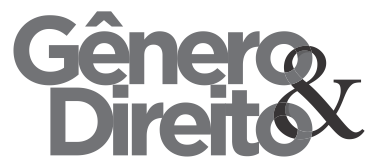

tomarse medidas para evitar su uso lesivo. Por ello, en 1948 se reconoció por primera vez el acceso a los avances científicos, en la Declaración Universal de los Derechos Humanos, como el derecho a "tomar parte libremente en la vida cultural de la comunidad, a gozar de las artes y a participar en el progreso científico y en los beneficios que de él resulten" (art. 27).

Este derecho surge y se dirige, por un lado, a la libertad en la generación de conocimiento científico, y, por el otro, a la garantía del acceso y beneficio de los avances científicos (Chapman, 2009). Con menos éxito, ${ }^{9}$ este derecho también tuvo vocación de constituir un límite en el desarrollo o la aplicación del tecnologías perjudiciales.

En todo caso, desde la Declaración se institucionalizó, al menos en los papeles, la voluntad de los Estados de fomentar y distribuir igualitariamente el avance científico. En esta línea, unos años después, el Pacto Internacional de Derechos Económicos, Sociales y Culturales (PIDESC) incorporó explícitamente el derecho de toda persona

${ }^{9}$ Este último punto es el menos desarrollado ya que se refiere, por ejemplo, a la creación de armas de
Cedico do Núcleo de Estudos e Pesquisas sobre Gênero e Direito

Centro de Ciências Jurídicas - Universidade Federal da Paraíba

V. 6 - No 03 - Ano 2017 (Spanish Edition)

252

a "gozar de los beneficios del progreso científico y de sus aplicaciones" (15.1.b). Y en 1975, en la Declaración sobre la utilización del progreso científico y tecnológico en interés de la paz y en beneficio de la humanidad, la ONU vinculó este derecho con el mantenimiento de la paz. Luego, en 1989, la UNESCO emitió la Declaración de Venecia que contiene la más amplia conceptualización hasta el momento del derecho a gozar de los beneficios científicos (Müller, 2010). Estableciendo que este derecho implica libertad tanto para la producción de conocimiento científico y tecnológico, como para usarlo y beneficiarse de sus avances, postula como principios fundamentales la igualdad y no discriminación, el acceso en condiciones de igualdad material, y beneficia especialmente a los grupos y a las personas más desaventajadas, y refiere a la igualdad de género, a la rendición de cuentas y a la participación.

Mas adelante, en la Conferencia sobre Población y Desarrollo en el Cairo (1994), episodio global clave en el reconocimiento de los derechos

destrucción masiva, asunto en el que, todavía, no hay acuerdo entre los países 
reproductivos, se recordó la obligación estatal de asegurar la disponibilidad, accesibilidad y aceptabilidad de insumos y técnicas necesarias en el ámbito de la reproducción.

Asimismo en 1999, al ritmo de los nuevos avances en genética, la UNESCO lanzó su Declaración sobre el genoma humano y los derechos humanos para la educación, la ciencia y la cultura, en la que sostuvo que los avances derivados del conocimiento del genoma humano deben estar disponibles para el beneficio de toda la humanidad. ${ }^{10}$

En 2001, representantes de más de cincuenta países firmaron la "Declaración sobre derechos humanos y práctica médica" en la que se incorporó la mirada de profesionales de la salud para dar cumplimiento a los estándares de acceso al derecho a la salud desarrollados en la Observación General Núm. 14 del Comité DESC y se reafirmó la importancia del personal sanitario en la difusión y garantía de los derechos humanos.
En 2012, el informe de la Relatora Especial sobre Derechos Culturales dijo respecto al derecho a la salud que su contenido normativo incluye la "asistencia especial y duradera a las personas y los grupos que hayan tomado parte en la actividad de investigación y reconocimiento de los mismos; acceso a una atención médica de calidad; suministro de nuevas modalidades $\mathrm{o}$ productos de diagnóstico y terapia obtenidos gracias a la investigación; apoyo a los servicios de salud; acceso a los conocimientos científicos y tecnológicos; e instalaciones y servicios destinados a crear capacidades en materia de investigación" (Comité de Derechos Humanos de las Naciones Unidas, 2012). También enfatizó que existe una “obligación implícita para los países en desarrollo de dar prioridad al desarrollo, importación y difusión de tecnologías simples y baratas que puedan mejorar la vida de las poblaciones marginadas" más aún cuando estas innovaciones son "esenciales para una vida digna". Y apuntó la importancia de garantizar a las

Biología, que reconoce como principio fundamental la prevalencia de los derechos y el bienestar de las personas sobre los intereses del desarrollo de las sociedades.
10 También, un año más tarde, se aprobó la Convención Europea sobre la Protección de los Derechos Humanos y la Dignidad de las Personas en Relación con las Aplicaciones de la Medicina y la 
mujeres, a las adolescentes y a las niñas el acceso a la información, a los procesos y a los productos científicos.

En el ámbito latinoamericano, la Declaración Americana de los Derechos y Deberes del Hombre de 1948 se convierte en el primer documento en reconocer el derecho al beneficio del avance de la ciencia: "toda persona tiene el derecho de participar en la vida cultural de la comunidad, gozar de las artes y disfrutar de los beneficios que resulten de los progresos intelectuales $y$ especialmente de los descubrimientos científicos" (art. 13).

Varios años después, también a nivel regional, el Protocolo Adicional a la Convención Americana Sobre Derechos Humanos en Materia de Derechos Económicos, Sociales y Culturales, o "Protocolo de San Salvador", reconoció explícitamente a este derecho:

"Los Estados partes (...) reconocen el derecho de toda persona a: b. Gozar de los beneficios del progreso científico y tecnológico. 2. Entre las medidas que los Estados Partes en el presente Protocolo deberán adoptar para asegurar el pleno ejercicio de este derecho figurarán las necesarias para la conservación, el desarrollo y la difusión de la ciencia, la cultura y el arte" (art. 14).
En este ámbito regional, el vínculo entre el ejercicio del derecho al beneficio del progreso científico y su interseccionalidad con los demás derechos humanos fue reconocido por la Corte Interamericana en el fallo "Artavia Murillo y otros ("Fecundación In Vitro") vs/ Costa Rica" (2012). Ahí, la Corte estableció la garantía del derecho al acceso al más alto y efectivo progreso científico como indispensable para el ejercicio de la autonomía reproductiva. También afirmó que "el derecho a la vida privada se relaciona con... el acceso a servicios de salud reproductiva, lo cual involucra el derecho de acceder a la tecnología médica necesaria para ejercer ese derecho". Finalmente, señaló, en consonancia con la Asamblea General de las Naciones Unidas, "la relación entre éste derecho [al goce de los beneficios del progreso científico] y la satisfacción de las necesidades materiales y espirituales de todos los sectores de la población" (ONU, 1975).

El derecho a la salud resulta un ejemplo paradigmático del vínculo entre los desarrollos científicos y las posibilidades de ejercicio de otro derecho humano. Al fin y al cabo, el acceso a los 
insumos sanitarios y nuevos desarrollos científicos suelen ser la única forma de realizar el derecho a la salud. Fue por ello que el Consejo Económico y Social de las Naciones Unidas (CESOC) en su Observación General Núm. 14 de 2000 indicó que los Estados deben procurar "las tecnologías pertinentes (...) contra las enfermedades infecciosas" para dar cumplimiento efectivo con el derecho a la salud. De igual forma, el Comité de Bioética de la UNESCO reconoció el rol de la ciencia y la tecnología en materia de salud en su Declaración Universal sobre Bioética y Derechos Humanos" (UNESCO, 2005) y diversos órganos de monitoreo de los derechos humanos han llamado a los Estados a proporcionar las "tecnologías apropiadas" para el cumplimiento del derecho a la salud.

Respecto a los derechos a la salud sexual y reproductiva, muy recientemente el Comité de Derechos Económicos Sociales y Culturales reconoció que debido a "numerosos obstáculos jurídicos, procedimentales, prácticos y sociales el acceso a todos los establecimientos, servicios, bienes e información en materia de salud sexual y reproductiva" (Comité DESC, 2016) el derecho a la salud, especialmente el de las mujeres, se restringe severamente. Y enfatizó la importancia de asegurar la disponibilidad de "personal médico y profesional capacitado y proveedores calificados que estén formados para prestar todos los servicios de atención de la salud sexual y reproductiva" así como de "medicamentos esenciales, incluida una amplia gama de métodos anticonceptivos, como los preservativos y los anticonceptivos de emergencia, medicamentos para la asistencia en casos de aborto y después del aborto" (Comité DESC, 2016). Afirmó a su vez que "[e]1 hecho de no incorporar o rechazar los avances y las innovaciones tecnológicas en la prestación de servicios de salud sexual y reproductiva, como los medicamentos en relación con el aborto...pone en peligro la calidad de la atención" (Comité DESC, 2016).

\section{LOS LÍMITES A LA DISCRECIONALIDAD DE LAS DECISIONES ESTATALES}

Los gobiernos latinoamericanos están eludiendo su responsabilidad de facilitar la disponibilidad (que incluye el 
registro como primer paso) y accesibilidad del misoprostol y de la mifepristona afectando la calidad de la atención sanitaria que reciben las mujeres, poniendo en riesgo a mujeres, limitando las posibilidades de atención de los profesionales y servicios de salud, entre otros efectos disvaliosos.

Las directrices que guían las políticas de medicamentos son variadas y responden, en el mejor de los casos, a las necesidades y prioridades de cada Estado. En general, en América Latina estas políticas, al menos en su texto, están orientadas a la adopción de medicamentos esenciales, la regulación sanitaria de medicamentos, la promoción del uso racional de medicamentos, la garantía de seguridad, eficacia y calidad, entre otras. Sin embargo, estas políticas también deben cumplir con obligaciones de respeto, protección y garantía de los derechos constitucionales y humanos. Este es el compromiso que asumen los Estados al incorporar estos derechos a sus constituciones y cuando ratifican tratados internacionales de derechos humanos. Y entre sus obligaciones, está la atención de la salud de manera progresiva, según los mejores estándares de la práctica médica y la evidencia científica. Éste es un límite a la discrecionalidad estatal.

De este modo, la desidia o resistencia de los gobiernos latinoamericanos en la aprobación del misoprostol y mifepristona no puede justificarse como una mera decisión política de oportunidad o conveniencia, pues tienen obligaciones con las mujeres que surgen de los derechos a beneficiarse de los avances científicos, la vida, la salud, entre otros, en un marco de no discriminación ¿Cuál sería entonces una justificación aceptable para esta inacción? Hay quienes podrían argumentar, ante el reclamo de pasividad frente a la falta de registro de estos fármacos, que los Estados tienen la potestad de evaluar que medicamentos ingresan al país. Por supuesto, los sistemas de salud deben tener la capacidad de discernir, es decir de evaluar las nuevas tecnologías pues no todo lo nuevo aporta y/o puede introducirse si se tiene en cuenta un sistema de salud lo más igualitario y sostenible posible (Gaviria, 2017). Pero justamente estamos frente a fármacos seguros, de alto costo-efectividad y eficaces para reducir muertes maternas y 
mejorar la salud reproductiva de las mujeres. La seguridad que reportan ambos fármacos supera otros métodos usados en la práctica médica, como la oxitocina para inducción del parto, laminarias para la maduración del cuello del útero, o la dilatación y curetaje para la atención del aborto.

Desde el punto de vista de la eficiencia sanitaria, resultan tecnologías costo-efectivas no sólo por su precio sino por sus condiciones simples de almacenamiento, transporte y distribución. Tomemos el caso del misoprostol. En primer lugar, su uso en servicios de salud reproductiva no requiere hospitalización o uso de quirófano, dada su forma de administración (oral, vaginal o sublingual) y sus efectos (FLASOG, 2013). Por estas cualidades es adecuado para abortos ambulatorios en las primeras semanas (OMS, 2012). También puede usarse en procedimientos quirúrgicos pues previene complicaciones como la hemorragia (Weeks y Faúndes, 2005; Figo, 2012; OMS, 2014). En segundo lugar, su estabilidad a temperatura ambiente lo hace fácilmente transportable (no requiere refrigeración), permitiendo su uso en centros de salud de atención primaria (Shaw, 2007; CLACAL, 2010). Más aun, el misoprostol es más eficiente $y$ efectivo que la oxitocina (el medicamento más usado en la prevención y tratamiento de la hemorragia posparto), que sí requiere refrigeración y administración intravenosa -condiciones no siempre disponibles en sitios con mayor vulnerabilidad geográfica, socioeconómica o institucional-. En tercer lugar, sus vías de administración favorecen una mayor aceptabilidad por parte de las mujeres (Lauferie et al., 2005; Marlow et al., 2016; Løkeland, 2014; Chung, 2016; Teal et al., 2009). Finalmente, su costo es aproximadamente cien veces menor que cualquiera otra prostaglandina (FLASOG, 2013).

En Ciudad de México, antes de la reforma legal, el costo promedio del tratamiento de complicaciones por aborto inseguro era de \$209 dólares por mujer. Tras la puesta en funcionamiento de los servicios de aborto legal, el costo de un aborto con medicamentos pasó en promedio a $\$ 98$ dólares (CLACAI-IPAS, 2010; Levin et al., 2009). Este método no sólo impactó en los costos sino que 
contribuyó a una disminución total de la mortalidad materna de un $56 \%$ en comparación con la década del 90 y un 28,6\% desde 2006 hasta 2015 (Secretaría de Salud de México, 2016). Mientras tanto, en las jurisdicciones donde no está registrado para usos obstétricos o en dosis apropiadas, o su disponibilidad está restringida, los costos son mucho más altos, convirtiendo a América Latina la región donde el misoprostol se obtiene a más alto precio (Fernández et al., 2009).

También se han alegado, como justificación de la inacción, dificultades que podría tener el Estado para regular su distribución (y un potencial uso para "el aborto ilegal"). Esta objeción resulta inaceptable pues traslada el peso de la (in)capacidad de controlar efectivamente la venta de un medicamento a las mujeres que se encuentran en los casos de aborto legal y que no podrían acceder al medicamento al que tienen derecho.

La valoración de los riesgos asociados a la mifepristona, pero especialmente al uso de ella, por ejemplo, se ha basado en una escala de valores diferente a la de otros medicamentos

11 Agradecemos a Laura Gil (Oriéntame, Bogotá) por acercarnos este argumento.
¿Cuál sería la razón para esta diferenciación? Cuesta saberlo. Por ejemplo, el uso del ibuprofeno tiene mayores contraindicaciones y más riesgos asociados que la mifepristona, sin embargo, su aprobación tardó pocos años desde su descubrimiento y desde entonces se mantuvo en sus condiciones laxas de venta y receta pese a los estudios que muestran sus riesgos y múltiples contraindicaciones. ${ }^{11}$ Del otro lado, todo lo referente a la mifepristona se ha sobredimensionado; en Estados Unidos se intentó rechazar su registro utilizando informes sobre cuatro muertes por sepsis pese a la falta de evidencia sobre su causalidad (Murray y Wooltorton, 2005). Y este es sólo un caso donde se han empleado reportes científicos de riesgos aislados para impedir el uso de un medicamento que tiene un perfil de seguridad sólido, que rara vez está contraindicado y que previene muchas más muertes de las que potencialmente podría producir. ${ }^{12}$ Los sesgos variados en las políticas de medicamentos.

$\mathrm{La}$ falta de registro del misoprostol y mifepristona también

\footnotetext{
${ }^{12}$ Agradecemos la información dada por Laura Gil.
} 
podría atribuirse al proceso de aprobación de fármacos, dado que muchos países el registro puede iniciarse sólo mediante la solicitud de un laboratorio farmacéutico interesado en su producción, distribución, o comercialización, o en todo caso la legitimación activa para la solicitud de registro es extremadamente restrictiva. En Canadá, por ejemplo, los laboratorios se abstuvieron por mucho tiempo de iniciar la solicitud de aprobación de la mifepristona argumentado sobreexigencias administrativas. En efecto, se alegaba que la renuencia de los laboratorios respondía a los requisitos más onerosos impuestos para la aprobación de medicamentos sobre salud sexual y reproductiva en comparación con Europa. Lo sucedido en Canadá no es atípico; las faltas de solicitudes suelen justificarse, al menos por las farmacéuticas, en los escasos incentivos dado el tamaño de algunos mercados nacionales y los costos políticos, legales y administrativos involucrados en el proceso de registro.

Frente a ese escenario, los gobiernos alegan impotencia, dado que las agencias de medicamentos, en general, no están facultadas para requerir a las farmacéuticas que soliciten el registro o amplíen las indicaciones, ni hacerlas ellas de manera oficiosa. Ello es así pues, en general los sistemas de medicamentos están diseñados bajo el principio de pasividad, destinado a garantizar independencia y transparencia de los procesos de revisión y aprobación de medicamentos.

Sin embargo, la adhesión ciega a este principio sin atender a sus efectos es contraria al derecho a la salud y por lo tanto al objetivo último que justifica la existencia de un sistema de salud al menos en los Estados de derecho occidentales contemporáneos.

De hecho, los programas de reducción de costos, priorización y acceso especial de medicamentos que se han llevado adelante en algunos países son un precedente para adoptar una actitud activa respecto al misoprostol y a la mifepristona (Erdman et al., 2008). Estos programas son excepciones a la regla de neutralidad de las agencias de medicamentos, lo que indica que ésta pasividad no es un rasgo intangible. Más aun, como comentan Erdman et al. (2006) sobre el caso de mifepristona en Canadá "las reformas regulatorias 


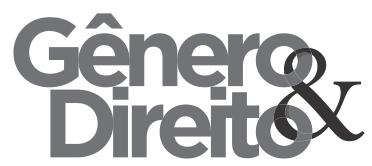

propuestas buscan expresamente

promover el compromiso temprano y cambiar los paradigmas regulatorios para ir de un sistema pasivo que es activado por los eventos en lugar de influir en los resultados".

Esta moderación en el principio de pasividad/neutralidad se vio en acción en el caso británico del medicamento Trastuzumab de Roche. A pesar de la abultada evidencia científica existente sobre la seguridad y efectividad para el tratamiento del cáncer de mama, el Reino Unido recién se decidió a registrar este fármaco cuando actores sociales y económicos reclamaron su aprobación e inclusión en el plan de salud público, lo que finalmente se hizo sin seguir el procedimiento estándar (Grecu, 2007).

Es decir, lo dicho aquí no es una idea descabellada menos aún exótica. Todo lo contrario, alrededor del mundo se pueden hallar ejemplos de medidas positivas adoptadas por autoridades sanitarias y de medicamentos destinadas a garantizar la disponibilidad de medicamentos, incluyendo el misoprostol y mifepristona. Concretamente, la intervención gubernamental facilitó la aprobación de la mifepristona en Francia, iódico do Núcleo de Estudos e Pesquisas sobre Gênero e Direito Centro de Ciências Jurídicas - Universidade Federal da Paraíba V. 6 - No 03 - Ano 2017 (Spanish Edition) 260 Suecia, Reino Unido y los Estados Unidos. Ya en 1988, y de manera pionera, el gobierno francés ordenó al titular de la patente de la mifepristona reanudar la comercialización y distribución de la mifepristona bajo amenaza de licencia obligatoria (Erdman et al., 2008), y "desde el momento en que el gobierno aprobó la droga, la mifepristona se convirtió en propiedad moral de las mujeres y no sólo en la propiedad de la compañía farmacéutica" dijo el Ministro de Salud francés tras ese episodio (Greenhouse, 2008).

También en América Latina hay unos pocos ejemplos que iluminan las posibilidades que tienen otros Estados aún renuentes a adoptar medidas activas y concretas. El caso colombiano muestra cómo las resistencias, incluso dentro del Estado, pueden trabajarse y superarse. Tras la aprobación por el Instituto Nacional de Vigilancia de Medicamentos (INVIMA) de algunos usos obstétricos del misoprostol, con la inclusión de la realización de abortos legales, la Procuraduría de la Nación puso en marcha una serie de acciones para torpedear el acceso al misoprostol, tanto dentro como fuera del sistema público de 
salud. Se solicitaron restricciones a su comercialización, medidas cautelares para impedir su distribución en los servicios públicos de salud, su exclusión del Plan Obligatorio de Salud (POS), entre otras. Finalmente, en 2012, un grupo de mujeres y organizaciones de la sociedad civil interpusieron una acción de tutela para que se ordenase a la Procuraduría poner fin a las obstrucciones en el ejercicio de los derechos reproductivos, a la información y al beneficio del progreso científico de las mujeres colombianas. El resultado fue la sentencia T- 627/2012 en la que la Corte Constitucional encontró que el Estado estaba obligado a garantizar el acceso efectivo a las tecnologías médicas más eficaces de acuerdo con la evidencia científica más actualizada, incluso mediante su inclusión en los seguros públicos de salud, y a sancionar cualquier obstrucción que impida el ejercicio de este derecho. Luego, en marzo de 2017, y también tras un arduo y larguísimo proceso con incidencia y aportes de la sociedad civil en trabajo con el Ministerio de Salud, se registró la mifepristona (INVIMA, 2017).
Desde el punto de vista jurídico, los Estados están obligados a respetar, proteger y garantizar el derecho a beneficiarse de los avances científicos en el ámbito de la salud reproductiva, como medio para respetar y asegurar el derecho a la vida, la salud, incluido los derechos reproductivos. La falta de medidas encaminadas al registro, disponibilidad y accesibilidades del misoprostol y mifepristona perjudican a un grupo específico, las mujeres en edad reproductiva, configurándose una discriminación basada en el sexo, fuertemente alimentada por sesgos sobre el aborto, y por tanto la sexualidad y roles de las mujeres.

Si bien entonces, todo Estado tiene la competencia para delinear su política de medicamentos y de salud, debe hacerlo basado en derechos y en razones públicas. Lo desarrollado hasta aquí indica que no hay razones de seguridad, eficacia ni costo-efectividad para insistir en una actitud de pasividad respecto a la indisponibilidad de estos medicamentos que pueden salvar vidas y mejorar la atención de la salud de las mujeres. 


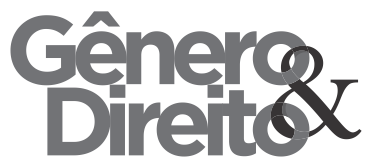

Todos estos derechos

mencionados suponen obligaciones, muy concretas, para los Estados latinoamericanos no solo porque han ratificado tratados internacionales de derechos humanos (que explícitamente reconocen el derecho a gozar de los avances científicos, que hablar del derecho a la vida, la salud y la no discriminación) sino porque, como ya apuntamos, la mayoría de sus constituciones nacionales también lo hacen.

Así, los Estados están obligados a adoptar las medidas necesarias, progresivas $\mathrm{y}$ no regresivas para garantizar el derecho a la salud. Parte de lo descripto en este artículo dejan entrever las consecuencias de la omisión estatal en adoptar medidas para promover el registro y disponibilidad del misoprostol y mifepristona.

La realización del más alto nivel posible de salud requiere que las mujeres tengan la libertad de decidir si y cuándo reproducirse, y el derecho a la atención de la salud necesario, lo que incluye acceder a técnicas e insumos para obtener una respuesta eficaz y de calidad. Y así surge del marco del PIDESC, que reconoce el iódico do Núcleo de Estudos e Pesquisas sobre Gênero e Direito Centro de Ciências Jurídicas - Universidade Federal da Paraíba V. 6 - No 03 - Ano 2017 (Spanish Edition) 262

derecho a la salud reproductiva incluidos bienes y servicios relacionados con el aborto que deben estar disponibles, accesibles, aceptables y de calidad. Más aún, el PIDESC impone la obligación de los Estados de asegurar la disponibilidad de medicamentos esenciales como una obligación fundamental derivada del derecho a la salud (Comité DESC, 2000). Esto supone que los gobiernos deben adoptar medidas, sean legislativas, administrativas, presupuestarias o de otra índole, para garantizar el acceso de las mujeres a los medicamentos esenciales de salud reproductiva.

La disponibilidad (que incluye la aprobación) y accesibilidad del misoprostol y la mifepristona se justifica también en razón del derecho a la igualdad (y no discriminación) en el ejercicio del derecho a beneficiarse de los avances científicos. Una carga importante para la salud de las mujeres está relacionada con la reproducción, y por eso la atención de la salud reproductiva ha sido identificada como una obligación prioritaria en virtud del derecho a la salud (Erdman et al., 2008).

La desidia y resistencia de los Estados para adoptar medidas concretas 
de pese a toda la evidencia y los datos de morbimortalidad materna, sugiere un comportamiento discriminatorio, basado en estereotipos y motivado por la percepción, irrelevantes normativamente, de que lxs funcionarixs públicos incluso empleadxs técnicxs pueden imponer sus preferencias morales, religiosas, y sus sesgos sexistas, incluso miedos políticos, en las políticas públicas destinadas a realizar derechos. En este sentido, el Relator Especial de las Naciones Unidas sobre el Derecho a la Salud ha recordado que el acceso a medicamentos para los grupos vulnerables no debe obstaculizarse por factores jurídicas o culturales, (OACDH, 2013). En especial, con relación al aborto, el Relator recuerda a los Estados el compromiso de eliminar todas las barreras de índole jurídica para el acceso a bienes, servicios e información, ya que pueden configurar una vulneración al derecho a la salud, así como las libertades y la dignidad de las personas afectadas.

No es necesario probar que los Estados tuvieron la intención de discriminar a las mujeres pues, como indica CEDAW la "discriminación contra la mujer" no sólo abarca toda distinción, exclusión o restricción basada en el sexo que tenga por objeto, sino también por resultado menoscabar o anular el reconocimiento, goce o ejercicio por la mujer. En el mismo sentido, se pronunció el Comité de Derechos Humanos en la “Observación general 18" (1989). El desarrollo del principio de no discriminación está previsto también en la CEDAW respecto a la igualdad en el acceso a la salud, que prevé la obligación de los Estados de examinar periódicamente la legislación en esta materia "a la luz de los conocimientos científicos y tecnológicos" para revisar, derogar o ampliarla según corresponda.

Por último, la falta de disponibilidad del misoprostol y de la mifepristona tiene un impacto desigual sobre determinados grupos de mujeres: afecta de modo diferencial en las mujeres según situación socioeconómica, geográfica, edad, etc. En consecuencia y como lo han venido diciendo desde la década del 90 las organizaciones rectoras de los consensos en salud global, la falta de perspectiva de género en la investigación en salud y las políticas sanitarias resulta profundamente "injusta porque priva a las mujeres, como clase de 
personas, de los beneficios de ese conocimiento" (CIOMS, 1993).

Esta injusticia no es nueva en materia reproductiva sino que ha sido forma reiterada en la investigación, aprobación y uso de medicamentos y tecnologías vinculadas a la sexualidad y reproducción de las mujeres (Mastroianni et al., 1994). Por ejemplo, en relación con la anticoncepción siempre se descuido la investigación de "métodos para hombres" (Sen et al., 2005) y se menospreciaron los efectos secundarios asociados a los métodos hormonales, o las infecciones derivadas del uso de los dispositivos intrauterinos (Germain et al., 1992), la aprobación de la píldora de emergencia fue retrasada en muchos países con fundamento en la protección de los preembriones (Bergallo, 2010). Estos sesgos tanto en la generación de evidencia, la práctica sanitaria y el diseño e implementación de las políticas públicas, muestran la incapacidad de los Estados para considerar y "separar los factores determinantes biológicos de los factores determinantes sociales de la desigualdad de género" (Sen et al., 2005) y dar un abordaje que permita tomar medidas de protección para las mujeres y otros grupos tradicionalmente discriminados en el campo de la salud.

\section{CONCLUSIONES}

El misoprostol y la mifepristona son tecnologías probadas, seguras, eficaces, costo-efectivas que pueden salvar la vida de mujeres y mejorar la atención de su salud, además de mejorar las experiencias de interrupción del embarazo. La falta de aprobación para usos obstétricos y otras barreras de disponibilidad y accesibilidad de estos medicamentos esenciales son una forma de discriminación, no nueva, hacia las mujeres, que los gobiernos latinoamericanos están obligados a remediar.

Las mujeres de América Latina siguen muriendo por hemorragia postparto y aborto inseguros pese a que hay formas simples de evitarlas. El misoprostol y la mifepristona son dos medicamentos que pueden justamente ayudar en esta tarea. De hecho, a la sombra y pese a la insolencia estatal, están ayudando -y podrían ayudar aún más- en la disminución de la morbimortalidad materna. 


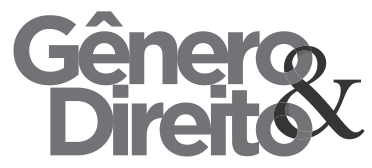

No sólo la muerte por causas asociadas al embarazo constituye una violación al derecho a la vida, así como la morbilidad materna al derecho a la salud sino que debe recodarse que la mayoría de los sistemas jurídicos de la región cuanta con abortos despenalizados al menos bajo el régimen de causales y por lo tanto hay obligaciones concretas por parte del Estado. En el mismo sentido, la atención de las complicaciones de aborto son servicio de salud básico incluso en aquellos lugares que penalizan, ya sea simbólica o efectivamente, el aborto. Estos medicamentos son insumos básicos para garantizar este servicio de salud.

La falta de aprobación y distribución de estos medicamentos ha restringido indebidamente su uso, aumentado su costo (en la región en donde el misoprostol se vende al precio más alto) y expuesto a muchas mujeres a falsificaciones o dosis inadecuadas.

La mayoría de los Estados latinoamericanos ha adoptado una posición indolente y sesgada, incluso reactiva, en al evaluación de estos dos fármacos. En efecto, las decisiones de las agencias de medicamentos y demás autoridades sanitarias de no impulsar la riódico do Núcleo de Estudos e Pesquisas sobre Gênero e Direito Centro de Ciências Jurídicas - Universidade Federal da Paraíba V. 6 - No 03 - Ano 2017 (Spanish Edition) 265 aprobación, de obstruir su accesibilidad y de no promover un uso más extendido del misoprostol y la mifepristona reflejan más posiciones morales particulares, conveniencias políticas, desconocimiento de obligaciones legales y desprecio hacia las mujeres (basado en estereotipos y estigmatizaciones) que posiciones basadas en la evidencia y en las obligaciones respecto a la salud de las mujeres, las condiciones de trabajo de los profesionales de la salud y un buen funcionamiento del sistema de salud.

Es decir, la falta de registro del misoprostol para uso obstétrico y de la mifepristona en esta parte del mundo no responde meramente a la falta de interés de los laboratorios. Los gobiernos no han adoptado medidas como sí lo hicieron con otros medicamentos, cuando han estimado que una política de medicamentos pasiva no era la repuesta adecuada. El margen de discreción con la que cuentan los gobiernos para adoptar políticas y garantizar el ejercicio de los derechos humanos no es ilimitado. Por el contrario, deben poder justificar públicamente sus elecciones y prioridades de política, y los motivos en que se basaron para adoptarlas. En los 


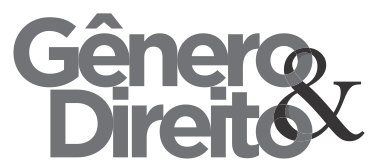

últimos años, se ha enfatizado la necesidad de políticas con un enfoque basado en derechos humanos, ello implica la participación de las personas interesadas, rendir cuentas sobre sus acciones y tomar las medidas necesarias para garantizar el ejercicio de los derechos. En el caso del misoprostol y de la mifepristona, los datos sobre los usos off-label en la región, la evidencia acerca de su eficacia, seguridad y aceptabilidad en aborto, y su incidencia positiva sobre la morbimortalidad deberían impulsar una actuación del Estado.

\section{REFERENCIAS BIBLIOGRÁFICAS}

Barbosa, Regina Maria; Arilha, Margarethe (1993), "The Brazilian Experience with Cytotec", en: Studies in Family Planning. Vol. 24, No 4. 1993, pp. 236-240.

Berer, Marge (2005), "Medical Abortion: Issues of Choice and Acceptability", en: Reproductive Health Matters. Vol. 13. 2005, pp. 25-34.

Bergallo, Paola. (2010), "El debate jurídico en torno a la anticoncepción de iódico do Núcleo de Estudos e Pesquisas sobre Gênero e Direito Centro de Ciências Jurídicas - Universidade Federal da Paraíba V. 6 - No 03 - Ano 2017 (Spanish Edition) 266

emergencia: una mirada comparada.".

São Paulo: Oficina Editorial, pp. 09-62.

(2014), The Struggle against Informal Rules on Abortion in Argentina. Abortion Laws in Transnational Perspective: Cases and Controversies.

Centro de Derechos reproductivos (2015), Aborto y Derechos Reproductivos en América Latina: implicaciones para la democracia. Consultado el 30 de noviembre de 2017 en

https://www.reproductiverights.org/sites/ crr.civicactions.net/files/documents/Abo rto-y-Derechos-Reproductivos-en-

America-Latina.pdf

Chapman, Audrey (2009), "Towards an Understanding of the Right to Enjoy the Benefits of Scientific Progress and its Applications", en: Journal of Human Rights. Vol. 8, No 1. pp. 1-36.

Chung, Ho Pak (2006), “Women's Perceptions on Medical Abortion", en: Contraception. Vol. 74, No 1. Julio, 2006, pp. 11-15. 


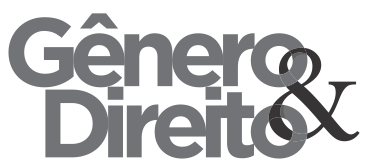

Council for International Organizations of Medical Sciences - CIOMS (1993), International ethical guidelines for biomedical research involving human subjects. Ginebra: Council for International Organizations of Medical Sciences.

Clarke, Adele; Montini Theresa (1993), "The Many Faces of RU486: Tales of Situated Knowledges and Technological Contestations", en Journal of Science, Technology and Human Values", articulo presentado en la American Society for Law and Medicine Conference, Arlington, VA, 6-7 Diciembre.

Consorcio Latinoamericano contra el Aborto Inseguro; Federación Latinoamericana de Sociedades de Obstetricia y Ginecología (2013), "Misoprostol: regulaciones y barreras en el acceso al aborto legal". Lima: CLACAI.

Consorcio Latinoamericano contra el Aborto Inseguro, (2012), "Misoprostol y aborto con medicamentos en
Ciódico do Núcleo de Estudos e Pesquisas sobre Gênero e Direito Centro de Ciências Jurídicas - Universidade Federal da Paraíba V. 6 - No 03 - Ano 2017 (Spanish Edition) 267

Latinoamérica y el Caribe". Lima:

\section{CLACAI}

Comité de Derechos Económicos, Sociales y Culturales (Comité DESC) (2000), "Comentario general 14: el derecho al más alto nivel posible de salud: 11/08/2000”. Suiza: 2000. $\mathrm{E} / \mathrm{C} 12 / 2000 / 4$. (2016), “Observación general 22: relativa al derecho a la salud sexual y reproductiva (art. 12 del Pacto Internacional de Derechos Económicos, Sociales y Culturales)". E/C.12/GC/22. 2 de mayo de 2016.

Consejo de Derechos Humanos (2012), Informe de la Relatora Especial sobre los derechos culturales, Farida Shaheed. A/HRC/20/26. Ginebra: ONU.

Coêlho, Helena Luna Lutéscia et al (1993), "Misoprostol and Illegal Abortion in Fortaleza, Brazil", en: The Lancet. Vol. 341, No 8.855. Mayo, 1993, pp. 1.261-1.263.

Erdman, Joanna et al (2008), "Medication Abortion in Canada: A 


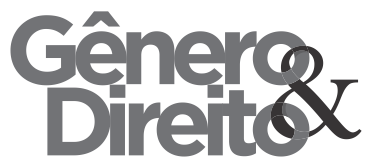

Right-to-Health Perspective", en:

American Journal of Public Health. Vol.

$98, \mathrm{~N}^{\mathrm{o}}$ 10. Octubre, 2008, pp. 1.764-

1.769 .

Faúndes, Aníbal (2007), “Introducción.

Uso de misoprostol en obstetricia y ginecología". $2^{\circ}$ ed. Ciudad de Panamá: pp. 8-16.

Faúndes, Aníbal; FIGO Working Group on the Prevention (2011), "The Combination of Mifepristone and Misoprostol for the Termination of Pregnancy", en: International Journal of Gynecology and Obstetrics. Vol. 115, N ${ }^{\circ}$ 1. Londres, 2011, pp. 1-4.

Federación Colombiana de Ginecología y Obstetricia (2013), “Concepto técnico: mifepristona”. Consultado el 08.07.2017 http://www.elespectador.com/noticias/sa lud/invima-aprueba-medicamento-masefectivo-y-seguro-para-interrupcion-delembarazo-articulo-682867

Federación Latinoamericana de Sociedades de Ginecología y Obstetricia (FLASOG) (2013), Uso del Misoprostol
Fernández, María et al (2009), "Assessing the Global Availability of Misoprostol", en: International Journal of Gynecology and Obstetrics. No 105. Londres, 2009, pp. 180-186.

Gaviria, Alejandro (2017), "Me da miedo que mis hijos no puedan crecer conmigo", Diario el Tiempo, 4.7.2017. Consultado el 08.07.2017 en http://www.eltiempo.com/vida/salud/alej andro-gaviria-ministro-de-salud-hablasobre-su-cancer-105236

Germain, A., Holmes, K.K., Piot, P., Wasserheit, J.N., eds. (1992). Reproductive tract infections: Global impact and priorities for women's reproductive health. New York: Plenum Press.

Greenhouse, Steven (1988), "France Ordering Company to Sell its Abortion Drug”, New York Times. 29 de octubre. Nueva York, 1988, A1. Citado por Erdman, Joanna el at (2008) op. cit. 


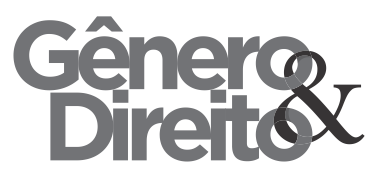

Periódico do Núcleo de Estudos e Pesquisas sobre Gênero e Direito Centro de Ciências Jurídicas - Universidade Federal da Paraíba V. 6 - No 03 - Ano 2017 (Spanish Edition) ISSN | 2179-7137 | http://periodicos.ufpb.br/ojs2/index.php/ged/index 269

Grecu, Eugen et al. (2007), "The Forced Adoption of a Fast-track Appraisal Process for a Breast Cancer Treatment in UK." IEMS Institut d'économie et management de la santé UNIL, Mémoire $n^{\circ} 105$.

Gruskin, Sofia et al. (2008), "Using human rights to improve maternal and neonatal health: history, connections and a proposed practical approach". Bulletin of the World Health Organization , 86, 589-593.

Guttmacher, Alan (2016), "Fertility of Man”, en: Fertility and Sterility. Vol.3, No 4, 2016, pp. 281-289.

Gynuity Health Projects (2014), "Health Projects: Misoprostol Aprobada" . Consultado el 08 de Julio de 2017 en http:/gynuity.org/resources/info/map-ofmisoprostol-approval-en/

------ (2013), "Venture Strategies Innovatios: Global Misoprostol Registration by Indication", 2. Consultado el 08 de julio de 2017 en http://gynuity.org/resources/info/map-ofmisoprostol-approval-en/
Harris, Lisa H; Daniel, Grossman (2011). "Confronting the challenge of unsafe second-trimester abortion." En International Journal of Gynecology \& Obstetrics 115.1: 77-79.

Health Canada (2006), "Blueprint for Renewal: Transforming Canada's Approach to Regulation Health Products and Food. Ottawa, Ontario". Consultado el 30 de noviembre de 2017 en http://www.hc-sc.gc.ca/ahc-asc/branchdirgen/hpfb-dgpsa/blueprint-plan/indexeng.php

Instituto Nacional de Vigilancia de Medicamentos y Alimentos (2017), "Invima aprueba medicamento más efectivo y seguro para interrupción de embarazo", 3 de marzo de 2017. Consultado el 08.07.2017 en http://www.elespectador.com/noticias/sa lud/invima-aprueba-medicamento-masefectivo-y-seguro-para-interrupcion-delembarazo-articulo-682867.

Kiley, Jessica W., et al (2010). "Delays in request for pregnancy termination: comparison of patients in the first and 


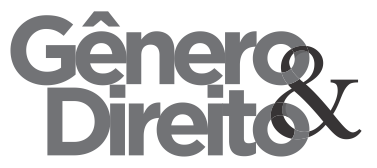

second trimesters." en Revista

Contraception 81.5: 446-451.

Lafaurie, María Mercedes et al (2009), "Women's Perspectives on Medical Abortion in Mexico, Colombia, Ecuador and Peru: A Qualitative Study", en: Reproductive Health Matters. Vol. 13, N 26. Londres, noviembre, 2005, pp. 75-83.

Lesbianas y feministas por la descriminalización del aborto (2015), Informe de la línea de atención "Más información, menos riesgo". Buenos Aires, 2015.

Levin, Carol et al (2009), "Exploring the Costs and Economic Consequences of Unsafe Abortion in Mexico City before Legalization”, en: Reproductive Health Matters. Vol. 17, N 33. Londres, 2009, pp. 120-132.

Løkeland, Mette et al (2014), "Medical Abortion with Mifepristone and Home Administration of Misoprostol up to 63 Days' Gestation", en: Acta Obstetricia et Gynecologica Scandinavica. Vol. 93, No
Ciódico do Núcleo de Estudos e Pesquisas sobre Gênero e Direito Centro de Ciências Jurídicas - Universidade Federal da Paraíba V. 6 - No 03 - Ano 2017 (Spanish Edition)

270

7. Gotemburgo, mayo, 2014, pp. 647653.

Marlow, Heather et al. (2016), "Women's Experiences with Medication for Menstrual Regulation in Bangladesh”, en: Culture, Health and Sexuality. Vol. 18, No 3. Sydney, 2016, pp. 349-360.

Mastroianni, Anna C; Ruth Faden; Daniel Federman (1994), Women and health research: Ethical and legal issues of including women in clinical studies. Washington, DC: Institute of Medicine, National Academy Press

Müller, Amrei (2010), "Remarks on the Venice Statement on the Right to Enjoy the Benefits of Scientific Progress and its Applications (Article $15 \quad$ (1)(b) ICESCR)", en: Human Rights Law Review. Vol. 10, No 4. 2010, pp. 765-784. Murray, Sally y Wooltorton, Eric (2005), "Septic Shock after Medical Abortions with Mifepristone (Mifeprex, RU 486) and Misoprostol", en: Canadian Medical Association Journal. Vol. 173, No 5. 2005, pp. 485-485. 


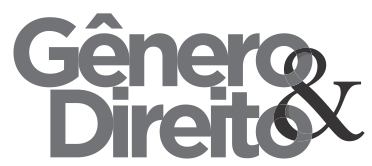

Organización Mundial de la Salud (OMS) (1986), "Maternal mortality: helping women off the road to death," 1986. 40, 175-183.

------- (2005), “Lista modelo de medicamentos esenciales". 14 ${ }^{\mathrm{a}}$ edición. Ginebra: OMS, marzo de 2005, p.22.

------- (2008), “Unsafe Abortion: Global and Regional Estimates of the Incidence of Unsafe Abortion and Associated Mortality in 2008". Ginebra: OMS.

------- (2011), Unsafe abortion: global and regional estimates of the incidence of unsafe abortion and associated mortality in 2008. Sexta edición. Ginebra: Departamento de Investigación en salud reproductiva, OMS.

------ (2015), Funciones del personal sanitario en la atención para un aborto sin riesgos y los métodos anticonceptivos después del aborto. Ginebra: OMS.

------- (2016), Prevención del aborto peligroso. Nota descriptiva mayo de 2016. Ginebra: OMS. eriódico do Núcleo de Estudos e Pesquisas sobre Gênero e Direito Centro de Ciências Jurídicas - Universidade Federal da Paraíba V. 6 - No 03 - Ano 2017 (Spanish Edition) 271

Organización de las Naciones Unidas (1975), “Declaración sobre la utilización del progreso científico y tecnológico en interés de la paz y en beneficio de la humanidad". Ginebra: ONU.

\section{(2013), "Report of the Special} Rapporteur on the Right of Everyone to the Enjoyment of the Highest Attainable Standard of Physical and Mental Health". Human Rights Council. Anand Grover, on access to medicines. A/HRC/23/42. Ginebra: Naciones Unidas, $1^{\circ}$ de mayo, 2013. Párrafo 45.

Ramos, Silvina et al. (2004), "Para que cada muerte materna importe." Ciudad de Buenos Aires: Centro de Estudios de Estado y Sociedad (CEDES) y Ministerio de Salud de la Nación.

Romero, Mariana; Ramón Michel, Agustina (2014), "Seguimiento de la CIPD en América Latina y el Caribe después de 2014”. Documento técnico. Articulación regional de organizaciones de la Sociedad Civil Hacia Cairo +20. Enero, 2014. 


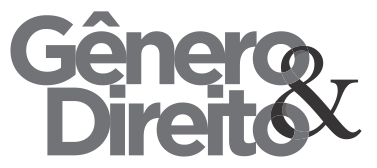

Jones, Bonnie Scott; Tracy A. Weitz (2009), "Legal barriers to secondtrimester abortion provision and public health consequences." en American Journal of Public Health 99.4: 623-630.

Secretaría de Salud de México (2016), "Informe Semanal de Vigilancia Epidemiológica. Defunciones Maternas”. Ciudad de México: Secretaría de salud de México.

Sen, Gita, Asha George, y Piroska Östlin (2005), "Incorporar la perspectiva de género en la equidad en salud: un análisis de la investigación y las políticas." Vol.14. Washington: Pan American Health Org.

Shaw, Dorothy (2007), "Misoprostol for Reproductive Health: Dosage Recommendations", en: International Journal of Gynecology and Obstetrics. $N^{\circ}$ 99. Londres, 2007, p. 155.

Távara Orozco, Luis et al. (2008), "Disponibilidad y uso obstétrico del misoprostol en los países de América Latina y el Caribe", Lima: Revista riódico do Núcleo de Estudos e Pesquisas sobre Gênero e Direito Centro de Ciências Jurídicas - Universidade Federal da Paraíba V. 6 - No 03 - Ano 2017 (Spanish Edition) 272
Peruana de Ginecología y Obstetricia 54.4 .

Távara Orozco, Luis; Chávez Alvarado, Susana (2013), "Regulación del uso obtétrico del misorpostol en los países de América Latina y el Caribe", en: Revista Peruana de Ginecología y Obstetricia. V.59 $\mathrm{N}^{\circ} 2$. Lima.

Teal, Stephanie et al. (2009), "Efficacy, Acceptability and Safety of Medication Abortion in Low-income, Urban Latina Women", en: Contraception. Vol. 80, No 5. Noviembre, 2009, pp. 479-483.

Organización de las Naciones Unidas para la Educación, la Ciencia y la Cultura -UNESCO- Asamblea General (2005), "Declaración Universal sobre Bioética y Derechos Humanos". París: UNESCO.

Fondo de las Naciones Unidas para la infancia -UNICEF (2011), "Estrategia de UNICEF en América Latina y el Caribe para contribuir a la morbimortalidad materna, neonatal e infantil". Nueva York: UNICEF. 
Winikoff, Beverly; Sheldon, William

Herbert (2012), "Use of Medicines

Changing the Face of Abortion", en:

International Perspectives on Sexual and

Reproductive Health. Vol. 38, N 3. 2012.

pp. 164-166.

Weeks, Andrew y Faúndes, Aníbal (2007), "Misoprostol in Obstetrics and Gynecology”, en: International Journal of Gynecology and Obstetrics. $\mathrm{N}^{\mathbf{0}} 99$. Londres, 2007, pp. 156-158; FIGO, op. cit. 2012; OMS, op. Cit. 2014.

Zamberlin, Nina et al (2012), “Latin American Women's Experiences with Medical Abortion in Settings where Abortion Is Legally Restricted", en: Reproductive Health. Vol. 9, No 34. Londres: Diciembre, 2012. 